
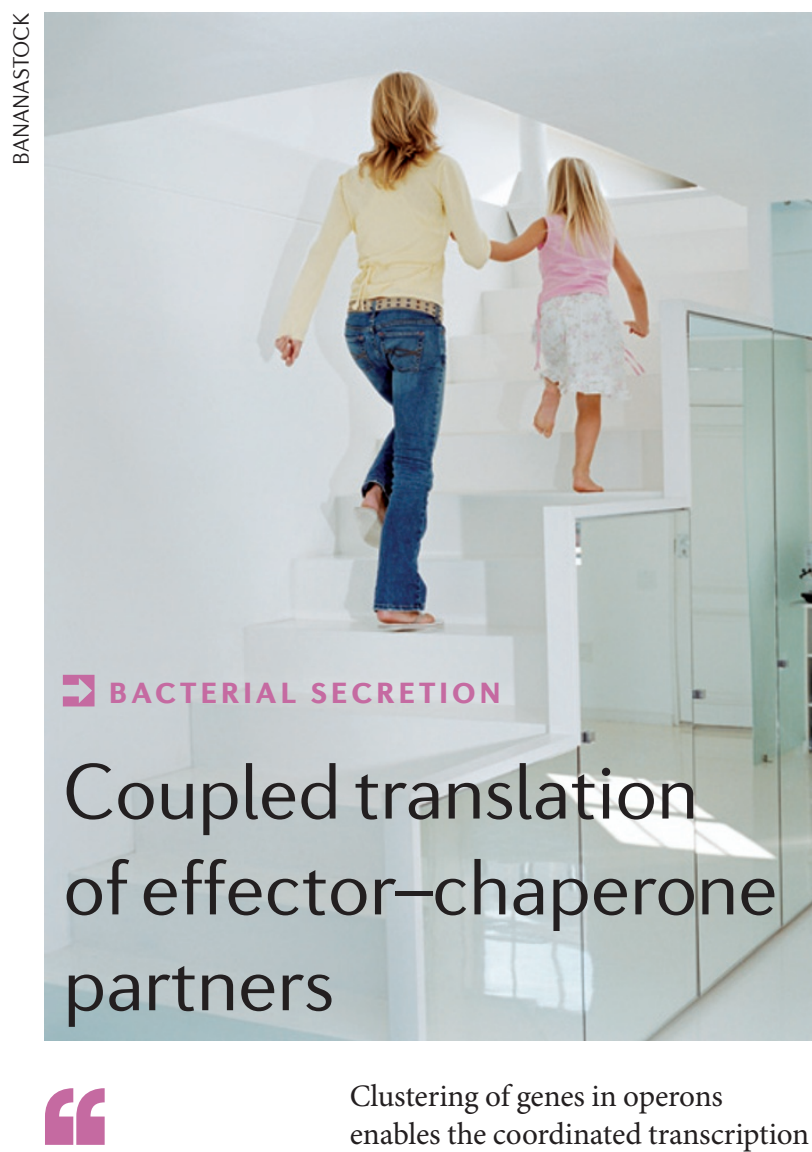

\section{whenever the} effector protein is translated, the chaperone will always be present in sufficient amounts to bind to the nascent effector and prevent it from folding prematurely or being degraded
Clustering of genes in operons enables the coordinated transcription of genes that encode functionally related products, so that these proteins are produced at the same time and in the same cellular location. Writing in Molecular Microbiology, Button and Galán now describe a type III secretion system (T3SS) effector and its cognate chaperone that are coupled not just at the level of transcription, but also during translation such that synthesis of the effector protein is dependent on the ongoing synthesis of its chaperone partner.

The T3SS is used by many Gram-negative bacteria to introduce effector proteins into eukaryotic host cells, where they modulate host responses to create a replicative niche for the bacteria. Proteins destined for secretion by this pathway interact with chaperone proteins that are thought to maintain the effectors in a secretioncompetent state. Some chaperones can bind to multiple effectors, whereas others are specific for a single effector. These 'uni-effector' chaperones are usually encoded adjacent to their cognate effectors, suggesting that expression of these proteins is transcriptionally coupled. For example, the gene encoding the Salmonella enterica subsp. enterica serovar Typhimurium effector SptP is immediately preceded by the gene that encodes its cognate chaperone, SicP. Interestingly, previous work had suggested that SptP and SicP are also coupled at a post-transcriptional level, as in the absence of SicP, not only is SptP rapidly degraded but also its expression is markedly reduced, despite the transcript level remaining unaffected. To investigate this further, Button and Galán replaced the chaperone-binding and catalytic domains of SptP with the reporter protein $\mathrm{PhoA}$, creating a chimaera that is not degraded in the absence of SicP. They introduced this fusion protein into strains of $S$. Typhimurium in which the sic $P$ coding sequence had been deleted $\left(\triangle \Delta_{i c} P\right)$ or disrupted by the introduction of a stop codon (sicPSTOP). In the $\triangle$ sicP strain, translation of the $s p t P-p h o A$ fusion was increased, whereas in the sicPSTOP strain, translation was slightly decreased. Importantly, neither expressing SicP in trans nor moving the sicP coding region to a position downstream of $s p t P-p h o A$ restored SptP-PhoA expression to wild-type levels, suggesting that it is not the SicP protein itself that is responsible for regulating the translation of SptP but rather the relative position of the sicP and sptP genes.

Using a series of deletion mutants, the authors mapped the region of the sicP gene that is responsible for regulating $s p t P$ translation to a region near the sptP start codon that was predicted to contribute to the formation of two stem loops in the transcript. Introducing mutations to destabilize these stem loops revealed that disrupting the downstream loop, which contained the $s p t P$ ribosome-binding site and start codon, had no effect on SptP synthesis but disrupting the upstream loop led to an increase in SptP levels. Furthermore, whereas introducing a stop codon into the sicP coding region decreased SptP-PhoA production, the introduction of a frame-shift mutation had no effect. Taken together, these data suggest that in the absence of ongoing SicP translation the stem loop in the sicP transcript can inhibit translation of SptP, but during SicP translation this regulatory stem loop becomes destabilized so that the ribosome can gain access to the sptP ribosome-binding site in the downstream stem loop.

What are the benefits of regulating the expression of functionally related proteins at both the transcriptional and translational levels? The authors suggest such coupling will ensure that whenever the effector protein is translated, the chaperone will always be present in sufficient amounts to bind to the nascent effector and prevent it from folding prematurely or being degraded.

Andrew Jermy

ORIGINAL RESEARCH PAPER

Button, J. E. \& Galán, J. E. Regulation of chaperone/ effector complex synthesis in a bacterial type III

secretion system. Mol. Microbiol. 4 Aug 2011

(doi: 10.1111/j.1365-2958.2011.07784.x) 